\title{
Research Paper: Comparing Tuberculin Anergy Skin Test Reactions and Lymphoblastic Transformation in Medical Students
}

\author{
Khosrow Agin $^{1^{*}}$, Mojtaba Ziaei ${ }^{1}$, Seid Davood Mansouri ${ }^{1}$
}

1. Loghman-e Hakim Hospital, School of Medicine, Shahid Beheshti University of Medical Sciences, Tehran, Iran.

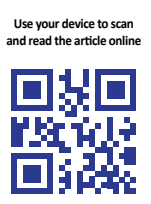

Citation: Agin Kh, Ziaei M, Mansouri SD. Comparing Tuberculin Anergy Skin Test Reactions and Lymphoblastic Transformation in Medical Students. International Journal of Medical Toxicology and Forensic Medicine. 2021; 11(2):32680. https://doi. org/10.32598/ijmtfm.v11i2.32680

https://doi.org/10.32598/ijmtfm.v11i2.32680

\section{Keywords:}

Bacillus Calmette Guerin (BCG) vaccine, Anergy, Tuberculin Skin test, Medical students, Iran

\begin{abstract}
A B S T RACT
Background: Tuberculin Skin Test (TST) reaction is an accepted screening test for diagnosing acute and latent Tuberculosis (TB) infection among at-risk populations. It is performed with a standard Protein Purified Derivative (PPD) solution. The skin reaction of PPD is unreliable to distinguish natural infection from the Bacillus Calmette Guerin (BCG) effect. The present survey aimed to determine the prevalence of Tuberculin Anergy (TA) reactions among medical students. Accordingly, we evaluated its sensitivity, specificity, and efficiency by applying the Recall Panel Antigen Test (RPAT) and the Lymphoblastic Transformation Test (LTT).

Methods: The participants were sequentially enrolled in this study according to the designed protocol. The current study was conducted on healthy medical students before registering for the hospital training course. All research subjects were healthy, having a cicatrix of Bacillus Calmette Guerin (BCG) vaccination on the arm, and at a young age (20-24 years).

Results: In total, 180 medical students met the inclusion criteria of the study. The study subjects' Mean \pm SD age was $22 \pm 3.07$ years (male $=89$, female=91). Moreover, $75(42 \%)$ subjects presented a TA reaction in the first step of TST screening. Furthermore, $13(64 \%)$ individuals presented a negative result of the RPAT. The sensitivity and specificity of the TST reactions, compared with the LTT were $100 \%$ and $94.4 \%$, respectively.

Conclusion: Significant TA reactions were found among medical students with positive cicatrix post-vaccinated. The obtained data suggested that the BCG protection for individuals may be decreased as time expired. Consequently, the medical students will especially be at high risk for TB infection during the clinical training course. The collected data highlighted the patient-safety from the viewpoint of forensic medicine.
\end{abstract}




\section{Introduction}

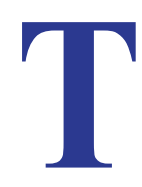

he incidence of Tuberculous (TB) infection was $13.7 \%$ per 100.00 among the Iranian population in 2013 [1]. The burden of $\mathrm{TB}$ in Iran was $6 \%$ in the eastern part of the Mediterranean region (2010) [2]. The trend of new cases of TB was increasing from 6.1 to 6.3 million from 2015 to 2016. Multidrug-Resistant strains were also raised in recent years worldwide [3]. Extensive evidence indicated that TB is a threat to individuals.

Subsequent involvement to latent $\mathrm{TB}$ and the emergence of the resistance of strains may be a diagnostic problem in healthcare staff and medical students $[4,5]$. Infected individuals may present mild or nonspecific symptoms and signs and lead to either failure or delayed diagnosis of TB [6]. The prevalence of undiagnosed TB was estimated to be $67 \%$ infected cases after death [7]. Furthermore, the prevention of infections among healthcare workers and the setting is an important point of patient safety [8] concerning mortality, morbidity, hospital stay, and cost [9]. Forensic medicine has a rule in the prevention and control of communicable diseases, medical liability, and health-related and protected problems.

The effective prevention strategy of TB transmission is based on the setting of an infection control program. It included detecting suspected and confirmed infected individuals, risk assessment, TB screening, environmental assessment, and risk classification on the number of beds [10].

Bacillus Calmette-Guérin (BCG) vaccine is the only universally-accepted vaccine against human Mycobacterium (MB) tuberculosis and is used since 1921. BCG plays a protective role concerning the mortality and morbidity among TB-infected populations.

The Iranian national policy documented a BCG vaccination protocol in infants. BCG produces a superficial ulcer after the intradermal injection. The healing process occurs several months post-vaccination and their cicatrix skin ulcer remains for life.

BCG skin cicatrix is on the upper arm representative as a post-vaccination sign. The protective effect of the $\mathrm{BCG}$ vaccine is variable and controversial in adolescences and adults [11]. The interval period of the protective effect of BCG takes almost 10 years [12] and can induce effector memory $\mathrm{T}$ cells [13]. The power of the current property of BCG is more related to previous TB infection, before vaccination, and sensitization to environmental MB [14].
The Tuberculin Skin Test (TST) reaction is a delayedtype hypersensitivity response. Purified Protein Derivative (PPD) is used as a material for performing the standard TST. It is a reliable screening test for diagnosing Latent Tuberculosis Infection (LTBI) and suspected TB with culture-negative MB among the at-risk population [15]. The prevalence of LTBI was reported to be $16 \%$ in Iranian healthcare workers [16]. Besides, the frequency of Iranian PPD positive was published among the general population and medical students as $26.2 \%$ and $38.9 \%$, respectively [15].

The anergy skin reaction is antigen-specific immunosuppression and can be generalized and cause false-negative TST reactions [17]. BCG vaccination may interfere with TST reaction interpretation. The current report suggested that the TST reaction provides a significant rate of negative values in previously young-vaccinated populations; it is associated with a high prevalence of TB infection in their countries [18]. Besides, PPD negative post-vaccinated healthcare workers had remained lower resistant to TB infection, compared to PPD positive subjects [19].

Tuberculin Anergy (TA) reaction is considered a health problem for high-risk groups, like medical students. Moreover, its reaction is unreliable for distinguishing natural infection from than BCG effect [20]. However, TA reaction depends on several factors, such as the strain and dose of the BCG vaccine, age, nutrition status, the number of years passed since vaccination, and the repeated TST [21].

The present survey aimed to determine the prevalence of TA reactions among medical students. We also evaluated its sensitivity, specificity, and efficiency by applying the Recall Panel Antigen Test (RPAT) and Lymphoblastic Transformation Test (LTT).

\section{Materials and Methods}

The present cross-sectional and descriptive was conducted in Loghman Hospital affiliated to Shahid Beheshti University of Medical Sciences (SBUMS).

The study sample was sequentially recruited among the first- and second-year students in the universities of medical sciences of Tehran and Shahid Beheshti. A total of 300 undergraduate medical students participated in the current study. Moreover, 180 medical students met the inclusion criteria of the study. All study participants provided written informed consent forms. 
The inclusion criteria included being undergraduate medical students with a history of BCG vaccination at birth; the presence of BCG cicatrix scar formation on the arm; being healthy; no history of past or current TB infection, and contact with identified TB-infected patients. Besides, the age, gender, as well as presence or absence of a BCG scar on the arm were considered as the inclusion criterion.

The exclusion criteria of the study were as follows: Active TB infection; Human Immunodeficiency Virus (HIV) infection; immunosuppressive diseases; a history of malnutrition; a history of viral infection one month before the study; repeated TST; malignancy, and undergoing corticosteroids. The skin cicatrix of BCG vaccination was classified as a real scar $(>2 \mathrm{~mm})$, tiny $(\leq 2 \mathrm{~mm})$, and not visible [22].

Initially, all selected participants conducted PPD standard test screening. The TST was performed with PPD, containing $0.1 \mathrm{~mL}$ of the solution of 5 Tuberculin Unit (TU). PPD was applied intradermally into the dorsal aspect of the left forearm using a plastic tuberculin syringe and a disposable $25 \mathrm{G}$ needle.

The test results were recorded 48-72 hours after the first injection. The obtained information was interpreted based on the presence of a skin induration. PPD skin reaction transversely measured the long axis of the forearm using the ballpoint method and was recorded in millimeters. The interpretation of PPD was instructed as per the Centers for Disease Control and Prevention (CDC) guideline. The skin indurations of PPD $<6 \mathrm{~mm}$ were defined specifically as negative; undetectable skin induration was determined as TA reaction.

TA subjects were selected in the second stage. The CDC recommended the additional use of $\geq 2$ control antigens with delayed-type hypersensitivity, including mumps antigen and tetanus toxoid. Anergy is defined as zero millimeters of skin induration for all delayed-type hypersensitivity antigens, i.e., administered in a given panel antigen. Skin tests with three recall antigens were administrated intradermal. Skin induration $>5$ millimeters was considered a positive response after 24 or 48 hours from the onset of injection. The healthy subjects consisted of skin reactions with $\geq 2$ antigens, and that an anergic subject has no response to any antigens.

LTT is the reflection of cell-mediated immunity and T cell function; as a rule, the LTT is reported to be wellcorrelated with a skin reaction. Next, the LTT was applied to the donors' peripheral lymphocytes. They have considered anergic if both PPD and panel skin test reac- tion results were negative. The lymphocytes were cultured in vitro with PPD and Phytohemagglutinin (PHA).

Furthermore, 9.5 ccs of a venous blood sample were obtained and mixed with $0.5 \mathrm{cc}$ heparin. It was incubated at 4C and immediately transferred to the immunology laboratory. Defibrinated whole blood was shaken with glass beads for $10 \mathrm{~min}$. Lymphoid cells are separated by centrifugation on a ficoll-isopaque gradient for 30 minutes at 2500 RPM. Centrifuged products and lymphocytes were collected at the interface by disinfected Pasteur pipette. The cells were washed three times at 1640 medium RPM. It was suspended at a concentration of $5 \times 10^{6}$ lymphocytes $/ \mathrm{mL}$ and was set up on flat-bottomed microplates.

The cells were stimulated with PPD and PHA solution antigens. Then, they were washed with PBS. It contains 2\% Autologous Plasma (AP). Lymphocytes were cultured in plastic tubes (polyethylene tube) in a volume of $1 \mathrm{~mL}$ of $10 \%$ human $\mathrm{AB}$ serum at 1640 medium RPMI. Antigens and mutagens (PPD, PHA) were added to the tubes in $0.1 \mathrm{~mL}$ volume. Its cultures were incubated for 2-8 days at $37^{\circ} \mathrm{C}$ within a $5 \% \mathrm{CO}_{2}$ atmosphere. It remained for 20 hours in the presence of $1 \mathrm{Ci}$ of $3 \mathrm{H}-$ labelled thymidines. The uptake of radioactivity was measured with a gamma counter after cell harvest on a semiautomatic harvester. The stimulation index was defined as follows: transformation (as counts per minute) in the presence of antigens/transformation in the absence of stimulants. Mean radioactivity is expressed as counts per minute (CT/min). It is determined using triplicate cultures by calculating the geometric mean [23].

Data analysis was performed in SPSS. The normality of the data was detected with the Kolmogorov-Smirnov test $(\mathrm{P}>0.5)$. The frequency was reported by percentage. Mean scores were performed using the Pearson correlation test, Chi-squared test, and Independent-Samples t-test. $\mathrm{P}<0.05$ was set as the significance level.

\section{Results}

One hundred and eighty undergraduate medical students were recruited in the current study. The study participants' Mean \pm SD age was $23.5 \pm 6.4$ years. In total, 92 females and 89 males participated in this study. BCG vaccination was performed in the birthing period, and the entire subjects had real skin BCG cicatrix. Of them, $78 \%$ had $>2 \mathrm{~mm}$ diameter, and $22 \%$ presented a tiny scar.

A total of 105 (58\%) subjects were positive skin tests for PPD. Of them, $58 \%$ were males and $42 \%$ females. Furthermore, $75(42 \%)$ of the studied students had TA 
Table 1. The results of stimulation indexes of PPD antigen and PHA mutagen

\begin{tabular}{|c|c|c|c|}
\hline \multirow{2}{*}{ Stimulation Indexs } & \multicolumn{2}{|c|}{ Mean $\pm S D$} & \multirow{2}{*}{$\mathbf{P}$} \\
\hline & Case & Control & \\
\hline PPD antigen & $1.4 \pm 0.54$ & $10.4 \pm 5.9$ & $<0.001$ \\
\hline PHA mutagen & $16.3 \pm 10.6$ & $15.2 \pm 4.8$ & $>0.05$ \\
\hline
\end{tabular}

skin reacted, (males $=37 \&$ females $=38$ ). No significant difference was found between gender and PPD responses $(\chi=1.36)$. Moreover, 47 subjects were enrolled from the TA skin reaction subset for the next step.

The RPAT was applied to the forearms of the TA skin reaction in the research subjects. Besides, 17 (36\%) participants indicated positive responses to RPAT. In total, 30 (64\%) students had negative RPAT responses (General Anergy; GA) (males=16, females=14).

A total of 17 subjects with negative RPAT entered in the next step for LTT as the gold standard detection test for true TA (males $=9 \&$ females $=8$ ). The results of the Stimulation Index (SI) are signified between the cases and controls for PPD antigen and PHA mutagen in Table 1. One female subject revealed a positive LLT.

The sensitivity and specificity of the TST reactions concerning LTT were $100 \%$ and $94.4 \%$, respectively. However, efficiency, Positive Predictive Value (PPV), and Negative Predictive Value (NPV) of the recent concept were reported to be $97 \%, 94 \%$, and $100 \%$, respectively. The TST and RPAT could detect true TA skin reactions among students with positive BCG skin scars.

\section{Discussion}

TB infection is the main causative human infectious source globally. The rate of infection is $100-300$ per 100000 in Asia. One-Third of the world's population is clinically-asymptomatic infected and known as latent TB. Eight million new cases of TB infection are found annually; $60 \%$ of those will die without treatment [24].

The World Health Organization (WHO) is the first organization that attempted the control and prevent the outbreak of TB worldwide. Mass BCG vaccination and case management are the accepted effective strategy suggested since August 1947 [25]. BCG vaccine is a living and weakened Mycobacterium Bovis strain, and was first used in 1912. BCG is the most popular vaccine and the current vaccine for protection against TB world- wide. Besides, its coverage efficacy is equal to $80 \%$. The frequencies of protective effect are estimated to be $50 \%$ overall and in serious infection are calculated as $78 \%$. It provides the most protective effect on TB in children (meningitis); it presents a variable efficacy in protection against pulmonary tuberculosis [26].

BCG vaccine may induce false-positive responses to the outcome of TST reactions. An essential causal factor that interferes with the BCG effect consists of the differences between BCG vaccines; the presence of the environmental mycobacteria; human genetics, and the differences of TB strains. The duration of BCG protection remains undiscovered; post-vaccination $\mathrm{BCG}$ reactivity has various responses concerning the TST reactions. It often persists for $<10$ years in the absence of exposed-M, $\mathrm{TB}$, and infection. Evidence revealed a protective effect of BCG against pulmonary TB lasting until 15 years [27]. Post-vaccinated protection efficacy BCG reactivity is up to $80 \%$ in children 11 . Its protective efficacy declines after 5 years and reaches $40 \%$ of the desired effect [28]. Our study findings disclosed a positive TST of 58\% among medical students, i.e., higher than the current report.

The prevalence of TA reactions is low in the healthy population $(0 \%-5 \%)$ [29]. The present study data indicated that the TA reactions were performed among hospitalized patients with a rate of $33.7 \%$ [30]. The study outcomes presented a $42 \%$ of TA reactions between focus populations.

\section{Conclusion}

Significant tuberculin anergy reactions were found among medical students with positive cicatrix postvaccination. The obtained study suggested that the BCG protection for individuals may be decreased as time expired. Consequently, the medical students will especially be at high risks for TB infection during the clinical training course. The resulting data is an important point in the patient-safety from the viewpoint of forensic medicine. 


\section{Ethical Considerations}

\section{Compliance with ethical guidelines}

All ethical principles were considered in this article. The participants were informed about the purpose of the research and its implementation stages; they were also assured about the confidentiality of their information; Moreover, They were allowed to leave the study whenever they wish, and if desired, the research results would be available to them. All authors read and accept the publication of the article.

\section{Funding}

This research did not receive any grant from funding agencies in the public, commercial, or non-profit sectors.

\section{Author's contributions}

All authors equally contributed to preparing this article.

\section{Conflict of interest}

The authors declared no conflicts of interest.

\section{Acknowledgements}

The authors want to thank the Tehran and Shaheed Beheshti Universities of Medical Sciences for completing the research project.

\section{References}

[1] Masoumi Asl H, Alborzi A, Pourabbas B, Kalani M. QuantiFERON-TB gold and tuberculin skin test for the diagnosis of latent tuberculosis infection in children. Iran J Med Sci. 2015; 40(5):4117. [PMID] [PMCID]

[2] Ebrahimi Kalan M, Yekrang Sis H, Kelkar V, Harrison SH, Goins GD, Asghari Jafarabadi M, et al. The identification of risk factors associated with patient and healthcare system delays in the treatment of tuberculosis in Tabriz, Iran. BMC Public Health. 2018; 18(1):174. [DOI:10.1186/s12889-018-5066-9] [PMID] [PMCID]

[3] Pradipta IS, Forsman LD, Bruchfeld J, Hak E, Alffenaar JW. Risk factors of multidrug-resistant tuberculosis: A global systematic review and meta-analysis. J Infect. 2018; 77(6):469-78. [DOI:10.1016/j.jinf.2018.10.004] [PMID]

[4] Sharma D, Sharma J, Deo N, Bisht D. Prevalence and risk factors of tuberculosis in developing countries through health care workers. Microb Pathog. 2018; 124:279-83. [DOI:10.1016/j.micpath.2018.08.057] [PMID]

[5] Kinikar A, Chandanwale A, Kadam D, Joshi S, Basavaraj A, Pardeshi G, et al. High risk for latent tuberculosis infection among medical residents and nursing students in India. PLoS One. 2019; 14(7):e0219131. [DOI:10.1371/journal. pone.0219131] [PMID] [PMCID]
[6] de Vries G, van Hunen R, Meerstadt-Rombach FS, van der Valk PDLPM, Vermue M, Keizer ST. Analysing tuberculosis cases among healthcare workers to inform infection control policy and practices. Infect Control Hosp Epidemiol. 2017; 38(8):976-82. [DOI:10.1017/ice.2017.100] [PMID]

[7] Stephenson L, Byard RW. An atlas overview of characteristic features of tuberculosis that may be encountered at autopsy. Forensic Sci Med Pathol. 2020; 16(1):143-51. [DOI:10.1007/s12024019-00161-y] [PMID]

[8] Di Paolo M, Papi L, Malacarne P, Gori F, Turillazzi E. Healthcare-associated infections: Not only a clinical burden, but forensic point of view. Curr Pharm Biotechnol. 2019; 20(8):658-64. [DO I:10.2174/1389201020666190618122649] [PMID]

[9] Siracusa M, Scuri S, Grappasonni I, Petrelli F. Healthcare acquired infections: Malpractice and litigation issues. Ann Ig. 2019; 31(5):496-506. [DOI:10.7416/ai.2019.2310 ] [PMID]

[10] Jensen PA, Lambert LA, Iademarco MF, Ridzon R, CDC Guidelines for preventing the transmission of mycobacterium tuberculosis in health-care settings, 2005. MMWR Recomm Rep. 2005; 54(RR-17):1-141. [PMID]

[11] No authors. The role of BCG vaccine in the prevention and control of tuberculosis in the United States. A joint statement by the advisory council for the elimination of tuberculosis and the advisory committee on immunization practices. MMWR Recomm Rep. 1996; 45(RR-4):1-18. https://pubmed.ncbi.nlm. nih.gov/8602127/

[12] Farhat M, Greenaway C, Pai M, Menzies D. False-positive tuberculin skin tests: What is the absolute effect of BCG and non-tuberculous mycobacteria? Int J Tuberc Lung Dis. 2006; 10(11):1192-204. [PMID]

[13] Zhu B, Dockrell HM, Ottenhoff THM, Evans TG, Zhang Y. Tuberculosis vaccines: Opportunities and challenges. Respirology. 2018; 23(4):359-68. [DOI:10.1111/resp.13245] [PMID]

[14] Roy A, Eisenhut M, Harris RJ, Rodrigues LC, SridharS, Habermann $S$, et al. Effect of BCG vaccination against mycobacterium tuberculosis infection in children: Systematic review and metaanalysis. BMJ. 2014; 349:g4643. [DOI:10.1136/bmj.g4643] [PMID] [PMCID]

[15] Rezai MS, Tabrizi R, Haghdoost AA, Afshari M, Abedi S, Akbari $\mathrm{M}$, et al. Estimating the prevalence of positive tuberculin skin test reactions in general population and high-risk groups: A meta-analysis. Int J Prev Med. 2017; 8:97. [DOI: 10.4103/ijpvm. IJPVM_140_17] [PMID] [PMCID]

[16] Mostafavi E, Nasehi M, Hashemi-Shahraki A, Esmaeili S, Ghaderi E, Sharafi S, et al. Comparison of the tuberculin skin test and the QuantiFERON-TB gold test in detecting latent tuberculosis in health care workers in Iran. Epidemiol Health. 2016 38:e2016032. [DOI:10.4178/epih.e2016032] [PMID] [PMCID]

[17] Pelly TF, Santillan CF, Gilman RH, Cabrera LZ, Garcia E, Vidal $\mathrm{C}$, et al. Tuberculosis skin testing, anergy and protein malnutrition in Peru. Int J Tuberc Lung Dis. 2005; 9(9):977-84. [PMID] [PMCID]

[18] Wang L, Turner MO, Elwood RK, Schulzer M, FitzGerald JM. A meta-analysis of the effect of Bacille Calmette Guerin vaccination on tuberculin skin test measurements. Thorax. 2002 57(9):804-9. https:// thorax.bmj.com/content/57/9/804.short

[19] Fjällbrant H, Ridell M, Larsson LO. The tuberculin skin test in relation to immunological in vitro reactions in BCG-vaccinated healthcare workers. Eur Respir J. 2001; 18(2):376-80. [DOI:10.11 83/09031936.01.00086901] [PMID] 
[20] Snider DE. Bacille Calmette-Guérin vaccinations and tuberculin skin test. JAMA 1985; 253(23):3438-9. [DOI:10.1001/ jama.1985.03350470090027]

[21] American thoracic society. Diagnostic standards classification of tuberculosis. Am Rev Respi Dis. 1990; 142(3):725-35. [DOI:10.1164/ajrccm/142.3.725] [PMID]

[22] No author. BCG vaccination. Br Med J (Clin Res Ed) . 1983 286(6368):876-7. https://www.ncbi.nlm.nih.gov/pmc/articles/PMC1547168/

[23] Smith JA, Reichman LB. Lymphocyte transformation. An aid in the diagnosis of tuberculosis in patients with nonreactive skin tests. Am Rev Respir Dis. 1972; 106(2):194-201. [DOI:10.1164/arrd.1972.106.2.194] [PMID]

[24] Smith I. Mycobacterium tuberculosis pathogenesis and molecular determinants of virulence. Clin Microbiol Rev. 2003 16(3):463-96. [DOI:10.1128/CMR.16.3.463-496.2003] [PMID] [PMCID]

[25] Raviglione MC, Pio A. Evolution of WHO policies for tuberculosis control, 1948-2001. Lancet. 2002; 359(9308):775-80. https://www.sciencedirect.com/science/article/abs/pii/ S0140673602078807

[26] Roy A, Eisenhut M, Harris RJ, Rodrigues LC, Sridhar S, Habermann S, et al. Effect of BCG vaccination against mycobacterium tuberculosis infection in children: Systematic review and meta-analysis. BMJ. 2014;349:g4643. [DOI:10.1136/ bmj.g4643] [PMID] [PMCID]

[27] Abubakar I, Pimpin L, Ariti C, Beynon R, Mangtani $\mathrm{P}$, Sterne JA, et al. Systematic review and meta-analysis of the current evidence on the duration of protection by bacillus Calmette-Guérin vaccination against tuberculosis. Health Technol Assess. 2013; 17(37):1-372, v-vi. [DOI:10.3310/ hta17370] [PMID] [PMCID]

[28] Nguipdop-Djomo P, Heldal E, Rodrigues LC, Abubakar I, Mangtani P. Duration of BCG protection against tuberculosis and change in effectiveness with time since vaccination in Norway: A retrospective population-based cohort study. Lancet Infect Dis. 2016; 16(2):219-26. [DOI:10.1016/ S1473-3099(15)00400-4]

[29] Zoloth SR, Safyer S, Rosen J, Michaels D, Alcabes P, Bellin E, Braslow C. Anergy compromises screening for tuberculosis in high-risk populations. Am J Public Health. 1993; 83(5):749-51. [DOI:10.2105/AJPH.83.5.749] [PMID] [PMCID]

[30] Woeltje KF, Kilo CM, Johnson K, Primack J, Fraser VJ. Tuberculin skin testing of hospitalized patients. Infect Control Hosp Epidemiol. 1997; 18(8):561-5. [DOI:10.2307/30141266] [PMID] 